\title{
A NOTE ON THE PHOTOLUMINESCENCE DECAY OF THERMALIZED EXCITONS IN QUANTUM STRUCTURES: MANY-BAND MODEL
}

\author{
D. S. Citrin* \\ Center for Ultrafast Optical Spectroscopy, The University of Michigan, \\ 2200 Bonisteel Boulevard, Ann Arbor, Michigan 48109-2099
}

(Received 5 August 1994 by J. Kuhl)

(accepted 26 Septeinber 1944)

\begin{abstract}
The temperature dependence of the photoluminescence decay time associated with the radiative decay of a thermalized exciton population in a quantum well or wire is shown to be independent of the number of exciton bands involved.
\end{abstract}

Keywords: A. Quentum Wells, D. Optical Properties, E. Luminescence, E. TimeResolved Optical Spectroscopies

Time-resolved photoluminescence (TRPL) spectroscopy with off-resonant excitation provides a probe into the radiative lifetimes of excitons in d-dimensional semiconductor heterostructures, such a quantum wells ( $Q W$ ) $(d=2)$ and quantum wires (QWR) $(d=1)$. It was realized quite early by Feldmenn, et af 1$]$. that the TRPL decay time has a temperature $(T)$ dependence $T(T)$ which also depends on $d$. For $Q W ' s$, the assumption of a thermal distribution of excitons occupjing a single parabolic band together with the requirement that only excitons with in-plane center-of-mass wavevector $\mathbf{k}_{\|}=\mathbf{0}$ can recombine radiatively (neglecting the photon momentum) leads to $T(T) \propto T$, which is indeed observed experiment- ally. $[1,2]$ Theoretical understanding of the TRPL decay time is refined by considering the radiztive decay of excitons within the context of polariton theory. $[3,4,5,6]$ Agranovich and Duborshii showed[4] that in $Q W ' s$ and QWR's only those excitons (i.e. excitonlike polaritons) with wavevectors smaller then the wavevector of light at the emission energy are iadiatively broadened; larger-wavevector states are stationary with respect to unassisted radiative decay. In addition to providing a self-consistent context for the description of excitonic radiative effects, the polariton theory also fixes the constant of proportionality in $T(T)$. Similar considerations for $Q W R^{\prime}$ give $r(T) \propto T^{1 / 2}$, which has very recently been confirmed by TRPL.|7] Thus, we can summarize the results for $Q W^{\prime}$ and $Q W R ' s$ as $\tau(T) \propto T^{d / 2}$.

The one-band model has been used to understand the $T$ dependence of the TRPL decay time in $Q W ' s$ since the work of Feldmann, et al. [1] It may be surprixing that this one-band model provides a quite accurate theoretical description over ather large temperature range, not only to give the $T$-dependence, but also the overall magnitude of the TRPL decay time, when in fact there are typically several exciton bands significantly popu-

[•] email: citrin Omich.physics.lsa.umich.edu. lated, especially at higher temperatures. One might therefore expect that as $T$ is raised and more and more bands become occupied, the optical dynamics will loose the characteristic $d$ dependence and increasingly resemble that of a bull aystem. We show here, howerer, that under fairly unrestrictive conditions this is not the case. Below it is shown that the many-band model for TRPL gives results closely resembling those obtained from the single-band model. $[5,6]$

We begin by stating our principle assumptions. (i) The homogeneous linewidth is assurned small compared with $\beta^{-1}=k_{\mathrm{B}} T$. At the same time, there is a scattering process operating which is rapid enough to maintain a thermal distribution of excitons while radiative decay is underway. This means that $T(T)$ is energyindependent. In addition, we neglect higher-order (e.g. phonon-assisted) and nonlinear processes. (ii) We take parabolic (direct) bands $j$, and denote the energy measured from the crystal ground state as $E_{j}+E_{j}^{\prime}=E_{j}+\frac{A^{2} k^{3}}{3 M_{j}}$ where $k$ is the magnitude of the excitation wayevector ( $k=k_{\|}$for the $\mathrm{QW}, k=k_{x}$ for the $\mathrm{QWR}$ ) and $M_{j}$ is the exciton mass. We shall assume $M_{j} \equiv M$ independint of $j$. (iii) Typically $M$ is sufficiently large and $E_{0}>\beta^{-1}$ so that the wavevector $\alpha$ at which band $j$ croses the !ight cone can be taken to be a constant $\alpha=E_{0} /(h c)$ where $c$ is the speed of light in the background dielectric medium. (iv) The oscillator ot rength of the radiative transition is independent of the exciton band involved. (v) $\beta^{-1}>\Delta$ where $\Delta=\frac{\hbar^{2} s^{2}}{2 M}$. (vi) We employ Boltemann statietics. (vii) We assume the temperature is sufficiently low so that we may neglect nonradiative recombination. (viii) The effect of static disorder is neglected. (ix) The confinement dimensions of the structure are assumed to be much less than the optical wavelength over $2 \pi$ in the medium of the emitted light.

The decay rate of the thermal exciton population is simply the average decay rate per exciton, 


$$
2 \Gamma(T)=\frac{A}{B},
$$

with

$$
\begin{aligned}
& A=\sum_{j} \int_{0}^{\infty} d E_{j}^{\prime} 2 \Gamma_{j}\left(E_{j}^{\prime}\right) e^{-\phi\left(E_{j}^{\prime}+E_{j}-E_{0}\right)} Q_{j}\left(E_{j}^{\prime}\right), \\
& B=\sum_{j} \int_{0}^{\infty} d E_{j}^{\prime} e^{-\phi\left(E_{j}^{\prime}+E_{j}-E_{0}\right)} e_{j}\left(E_{j}^{\prime}\right)
\end{aligned}
$$

where $e_{j}$ is the density of atates for exciton band $j$ and $\Gamma_{j}\left(E_{j}^{\prime}\right)=\Gamma_{j}(k)$ is the $k$-dependent decay rate (for band j) given in Ref. [6] for QW's and Ref. [8] for QWR's. The TRPL decay time is then $r(T)=[2 \Gamma(T)]^{-1}$. We use the convention that quantities $I$ refer to the decay rate of the wavefunction amplitude, hence the factor of 2 to describe population decay rates. Assumption (iii) above has as a consequence that $\rho_{j} \equiv Q$ independent of $j$. Since we are dealing with a thermal population, we can assume that the rates $\Gamma_{j}\left(E_{j}^{\prime}\right)$ have been averaged over degenerate exciton spin states (polarization). [6] Assumptions (iii), (iv), and (v) allow us to write

$$
\Gamma_{j}\left(E_{j}^{\prime}\right)=\Gamma \theta\left(\Delta-E_{j}^{\prime}\right) \text {. }
$$

The decoupling of the sum and the integral in Eq. (2) results, and the remaining sums in Eqs. (2) and (3) cancel. We then can evaluate Eqs. (1), (2), and (3) to give

$$
2 \Gamma(T)=2 \bar{\Gamma}\left(\frac{\Delta}{k_{\mathrm{B}} T}\right)^{d / 2} \pi^{(d-2) / 2} \quad d=1,2
$$

independent of the number of exciton bands. In particular, this is the result obtained in the one-band model[5, $6,8]$ as given above in the introductory paragraph.

It may be supposed that the conditions (i)-(ix) we invoke are rather restrictive. Let us consider them one by one. (i) We make no attempt to present a critical analyas of the assumption of a thermalized exciton distribution, whether within a single band or many bands. Nevertheless, since except at very lor temperature $(\sim 4 \mathrm{~K})$ in resonent experiments dephasing times are short compared with radiative decay times, it seems likely that the exciton population is at least approximately thermal. Moreover, the measured dependences $r(T) \propto T^{d / 2}$ and the foregoing analysis strongly support the validity of this assumption. (ii) Band-structure ef- fects are expected to modify the details of the picture somewhat, but not drastically. (iii) For semiconductors with a direct band gap in the optical range, $E_{0} \approx 1$ $\mathrm{eV}$, whereas $\beta^{-1}=26 \mathrm{meV}$ at room temperature. (iv) Once the decay rates are spin averaged, $\Gamma\left(h_{\|}\right)$for lighthole and heavy-hole excitons are comparable. Similar remarks apply to QWR's. Provided the spectral density of dipole-forbidden bands is roughly proportional to that of dipole-allowed bands, the precence of dipole-fobidden bands will simply reduce the effective value of $\bar{\Gamma}$. (v) For example, for GaAs-based structures, this assumption means $T \gg 1 \mathrm{~K} .[5,6]$ (vi) The validity of assumptions (v) and (vii) validates the use of classical statistics. (vii) For GaAs/AlGaAs QW's this essumption implies $T<80 \mathrm{~K}$, although there appears to be considerable sample dependence to the temperature above which nonradiative processes dominate.[2] (viii) Although localized states are expected to play a role even for $\beta^{-1}$ greater than the Stokes shift between luminescence and excitation spectra, $[6]$ the overall effect is to change the constant of proportionality in $T(T) \propto T^{d / 2}$ above some critical temperature. (ix) Again for GaAs-bused structures, this implies that confinement dimensions must be less then $x^{-1} \approx \mathbf{4 0 0} \AA$.

In summary, we have shown why the one-band model yields remarkably good results for the TRPL decay time despite the significant occupation of several exciton bands. We do not claim that the model, whether in its one- or many-band form, is adequate to fully describe TRPL experiments; we merely point out the surprising result that the andysis based upon the model is independent of the number of participating exciton bands. The same conclusion is reached both for $Q W ' s$ and for QWR's. In particular, $T(T)$ retains its unique signature of $d$ even when several exciton bands are signifcantly occupied. The assumptions (i)-(ix) were shown to be not overly restrictive for off-resonant TRPL within a particular temperature regime, although we do expect weak departures from the predicted $T$-dependence when one includes the realiatic band structure and distribution functions.

We are indebted to $A$. Forchel for suggerting the topic of this study. This work was supported by the National Science Foundation through the Fellows Program of the Center for Ultrafast Optical Science under STC PHY 8920108.

\section{References}

[1] J. Feldmann, G. Peter, E. O. Göbel, P. Dawson, K. Moore, C. Foxon, and R. J. Elliot, Phys. Rev. Lett. 50, 2337 (1987).

(2) M. Colocci, M. Gurioli, A. Vinattieri, F. Fermi, C. Deparis, J. Massies, and G. Neu, Europhys. Lett. 12, 417 (1990).

(3) J. J. Hopfield, Phys. Rev. 112, 1555 (1958).

4) V. M. Agranovich and A. O. Dubovekii, Pis'ma Zh. Eksp. Teor. Fiz. 3, 345 (1966) [JETP Lett. 3, 223 (1966)].
[5] L. C. Andreani and F. Bassani, Phys. Rev. B 41, 7536 (1990); L. C. Andreani, F. Tassone, and F. Bassani, Solid State Commun. 77, 641 (1991).

[6] D. S. Citrin, Phys. Rev. B 47, 3832 (1993).

77 H. Akiyama, S. Koshiba, T. Someyz, K. Wada, H. Noge, Y. Natamura, T. Inoshita, A. Shimizu, and H. Sakaki, Phys. Rev. Lett. 72, 924 (1994).

[8] D. S. Citrin, Phys. Rev. Lett. 69, 3393 (1992). 